BNL 66763

\title{
Resonant Inelastic X-ray Scattering from Transition Metal Oxides
}

\author{
J.P. Hill \\ Department of Physics, Brookhaven National Laboratory, Upton, NY $119^{7} 3^{1}$
}

\begin{abstract}
Recent developments in hard x-ray resonant inelastic x-ray scattering as a probe of strongly correlated systems are reviewed. Particular attention is paid to studies of $\mathrm{Nd}_{2} \mathrm{CuO}_{4}$. A charge transfer excitation is observed when the incident photon energy is tuned in the vicinity of the copper K-edge. It is shown that the presence of resonant enhancements is controlled by the polarization dependence of the excitation process and by the overlap between a given intermediate state and the particular excitation being studied. This latter observation has shed light on the non-local effects present in certain intermediate states.
\end{abstract}

\section{INTRODUCTION}

Strongly correlated electron systems $\Gamma$ and in particular the transition metal oxides $\Gamma$ are amongst the most actively investigated systems in condensed matter physics today. This interest is driven both by the wide variety of phenomena displayed by these materials and by the fundamental issues raised in trying to understand them. Notable examples in this class include $\Gamma$ the high- $T_{C}$ superconductors and the colossal magnetoresistance manganates.

Strongly correlated systems are characterized by electronic behavior intermediate between the highly localized behavior of ionic insulators and the completely delocalized behavior of simple metals and as a result a complete theoretical description remains problematic. The strong correlations preclude the possibility of successful band structure calculations $\Gamma$ and a variety of numerical approaches have therefore been applied $\Gamma$ utilizing small clusters of ions for which on-site interactions can be treated explicitly. These models are typically local $\Gamma$ that is the translational symmetry of the lattice is neglected. However $\Gamma$ as is increasingly recognized $\Gamma$ solid state (non-local) effects can be important and recently calculations have been performed for a number of clusters for which some degree of translational symmetry has been restored.

1) Work supported by the US D.O.E. under contract no. DE-AC02-98CH10886 
On the experimental front of crucial importance are measurements of the electronic structure and dynamics $\Gamma$ to provide insights into the behavior and to test the various theoretical approaches. In this endeavor $\Gamma$ inelastic x-ray scattering in the hard x-ray regime offers a number of potential advantages over other techniques. In particular Tit probes bulk-like properties and is a "photon-inTphoton-out" process. This ensures that the experiments do not suffer from so-called final state effects $\Gamma$ in which the interpretation of the observed spectra is complicated by the presence of a core-hole (as is the case in $\Gamma$ for example $\Gamma$ a photoemission process). In addition $\Gamma$ the technique can be applied to insulators $\Gamma$ without problems due to electrostatic charging $\Gamma$ and in principle experiments can also be performed in applied magnetic and electric fields.

In the non-resonant regime $\Gamma$ far from any absorption edges $\Gamma$ the cross-section is well understood and is given by the dynamic structure factor $\Gamma \mathrm{S}(\mathbf{q}, \omega)$. This quantity is proportional to the Fourier transform of the charge density-density correlation function and may be directly related to important quantities $\Gamma$ such as the complex dielectric function [1].

Unfortunately to date this technique has been limited largely to studies of low$\mathrm{Z}$ materials and is thus not applicable to the majority of interesting transition metal oxides. This is because the inelastic cross-section is extremely small and only by studying samples for which the x-ray absorption length is large resulting in relatively large sample volumes $\Gamma$ can this limitation be overcome and reasonable signals be obtained.

Recently $\Gamma$ however $\Gamma \mathrm{Kao}$ and co-workers discovered resonant enhancements in the inelastic scattering cross-section which offer the potential for overcoming this limitation [2] $\Gamma$ and it is these enhancements which are the subject of this review. Note this discovery of enhancements in the inelastic scattering of valence band excitations is in a certain sense an extension of the more familiar resonant Raman scattering from emission lines (see e.g. ref. [3]) to very small energy transfers.

Kao et al. observed the large increase in the inelastic scattering in $\mathrm{NiO}$ when the incident x-ray energy was tuned through the Ni K-edge. In particular $\Gamma$ they found excitations at $5 \mathrm{eV}$ and at $8 \mathrm{eV}$ that were only observable when the incident photon energy was tuned to the vicinity of the Ni K-edge. The intensity of each was found to be strongly dependent on the incident energy (while the position of the excitations moved only slightly with incident energy). Figure 1 shows this incident energy dependence for the $5 \mathrm{eV}$ excitation. A large number of sharp resonances are observed in the scattered intensity $\Gamma$ each of which appears to correspond to a feature in the absorption spectrum (solid line) Talbeit better resolved in the inelastic scattering.

On the basis of a configuration-interaction cluster model calculation KKao et al. identified these features as charge transfer-type excitations in which the system is excited from a $3 \mathrm{~d}^{8}$ ground state to a $3 \mathrm{~d}^{9} \underline{\mathrm{L}}$ excited state $\Gamma$ where the $\underline{\mathrm{L}}$ represents a hole on the ligand site. Of perhaps greatest significance was the size of the resonant enhancement. By comparing non-resonant x-ray data to electron scattering data taken at the same momentum transfer $\Gamma$ Kao et al. estimated that the resonant 
inelastic cross-section to be about 100 times larger than the non-resonant crosssection.

While a number of questions concerning the detailed understanding of the resonant cross-section arose as a result of the $\mathrm{NiO}$ work (and still remain to some extent) $\Gamma$ the potential for expanding the applicability of inelastic x-ray scattering to materials containing heavy elements $\Gamma$ and specifically the high- $\mathrm{T}_{C}$ cuprates $\Gamma$ was immediately apparent. Furthermore $\Gamma$ the technique offers the added advantage of element specificity allowing $\Gamma$ for example Tonly those excitations associated with the copper orbitals to be probed by tuning to the $\mathrm{Cu}$ K-edge.

Note that this new work in the hard x-ray regime extends and complements a larger body of work utilizing resonant inelastic scattering in the soft x-ray regime. This latter effort includes the early work of Ma and others (see eg. [45]) in studies of diamond $\Gamma$ graphite and other systems and extends to the recent work on strongly correlated systems (e.g. [6-9] and references therein). Theoretical efforts have accompanied these advances in soft x-ray techniques and include [10-12] and references therein.

In this paper $\Gamma$ we review a series of experiments carried out to elucidate the details of the resonant inelastic cross-section. These experiments $\Gamma$ primarily carried out on $\mathrm{Nd}_{2} \mathrm{CuO}_{4}$ observed a similar charge transfer process to that seen in $\mathrm{NiO \Gamma}$ and demonstrate the feasibility of using these techniques to study the high- $\mathrm{T}_{C}$

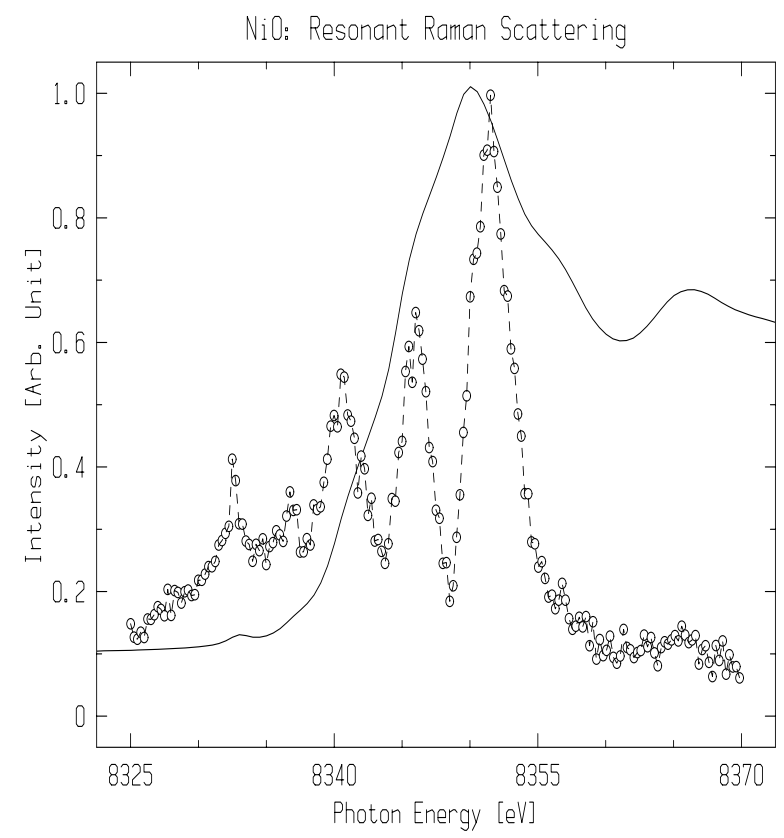

FIGURE 1. Incident photon energy dependence of a $5 \mathrm{eV}$ charge transfer excitations, as observed in $\mathrm{NiO}$, with the incident energy being tuned through the Ni K-edge (open circles). The solid line shows the Ni K-edge absorption spectrum for comparison. Figure taken from ref. [2]. 
cuprates. Measurements of the incident energy and polarization dependence of the cross-section illustrate the importance of the intermediate (highly excited) states in determining the resonant lineshape $\Gamma$ and in particular the sensitivity of that lineshape to non-local effects in the intermediate state. In addition $\Gamma$ the momentum dependence of the cross-section will be briefly discussed. Portions of this work have appeared previously [13-16].

\section{RESONANT INELASTIC SCATTERING IN $\mathrm{ND}_{2} \mathrm{CUO}_{4}$}

$\mathrm{Nd}_{2} \mathrm{CuO}_{4}$ is the parent compound of the electron doped high- $\mathrm{T}_{C}$ family $\mathrm{Nd}_{2-x} \mathrm{Ce}_{x} \mathrm{CuO}_{4}$. Its crystal structure is body centered tetragonal of the $T^{\prime}$ type and consists of $\mathrm{CuO}_{4}$ plaquettes connected in a two dimensional corner-sharing network. Unlike the $\mathrm{La}_{2} \mathrm{CuO}_{4}$ series $\Gamma$ there are no apical oxygens. The crystal used in this work was of high crystallographic quality (mosaic 0.014 ${ }^{\circ} \mathrm{FWHM}$ ) $\Gamma$ which minimizes the elastic scattering background $\Gamma$ and was in the form of a platelet $\Gamma$ $20 \mathrm{~mm} \times 10 \mathrm{~mm} \times 0.1 \mathrm{~mm}$ with a c-axis surface normal.

\section{A Experimental Details}

The $\mathrm{Nd}_{2} \mathrm{CuO}_{4}$ experiments were performed at two wiggler sources at the National Synchrotron Light Source $\Gamma$ beamlines X21 and X25. The initial work [13] was performed at X21 and utilized a four bounce monochromator comprised of two channel cut $\mathrm{Si}(220)$ crystals. The incident resolution was $0.2 \mathrm{eV}$ and the flux delivered on the sample was $5 \times 10^{10}$ photons $\mathrm{s}^{-1}$. The horizontally scattered radiation was collected by a spherically bent $\mathrm{Si}(553)$ analyzer and the overall energy resolution was a Gaussian of $1.9 \mathrm{eV}$ full-width-at-half-maximum (FWHM) Tas measured by the quasi-elastic scattering from the sample. The sample was oriented such that the momentum transfer was along the c-axis and the incident polarization was therefore largely perpendicular to the copper oxide planes [13]. A second set of measurements were performed at X25 ffor which a vertical scattering geometry was utilized [15]. In this case $\Gamma$ with the momentum transfer again along the $\hat{c}$-axis $\Gamma$ the incident polarization was entirely within the plane of the $\mathrm{CuO}$ sheets. and the $1 s \rightarrow 4 p_{\sigma}$ transition was utilized. For these latter measurements $\Gamma$ a double crystal $\mathrm{Si}(111)$ monochromator was used which provided an incident energy resolution of $2.2 \mathrm{eV}(\mathrm{FWHM})$ and delivered a flux of $5 \times 10^{11}$ photons $\mathrm{s}^{-1}$. The same analyzer set-up as in the X21 experiments was used $\Gamma$ and the overall energy resolution was $2.3 \mathrm{eV}$ (FWHM). All results discussed here were taken at room temperature at a momentum transfer of $q=4.6 \AA^{-1}$. The same sample was used in all measurements.

The experiments on $\mathrm{CuGeO}_{3}$ [14П17] were performed on X21 with an identical set-up to that outlined above. 


\section{B Charge Transfer Excitation}

The scattered intensity observed in $\mathrm{Nd}_{2} \mathrm{CuO}_{4}$ is plotted as a function of energy loss $\left(E_{i}-E_{f}\right)$ for a series of incident photon energies around the $\mathrm{Cu}$ K-edge in figure 2. Each data set took approximately 24 hours to collect. The excitation at $6 \mathrm{eV}$ is observed only for incident energies around 8990 eV Tand remains at approximately fixed energy loss for all incident energies.

The amplitude of the $6 \mathrm{eV}$ excitation was extracted by fitting the energy loss scans to a Gaussian peak on a sloping background (to account for the tails of the nearby $K \beta_{5}$ emission line) and the results are plotted in figure 3 as a function of incident photon energy. Resonant behavior was observed $\Gamma$ with a peak at $8990 \mathrm{eV}$. Even at resonance $\Gamma$ the count rates remain small $\Gamma$ due to the large absorption of the $\mathrm{Nd} \Gamma$ as well as that of the copper. Count rates were on order of a few counts per minute.

Also shown in figure 3 is the absorption of $\mathrm{Nd}_{2} \mathrm{CuO}_{4}$ powder $\Gamma$ in which features were observed at 8983Г8990Г8995 Гand $9002 \mathrm{eV}$ (see also refs. [19L20]). These have been interpreted as arising from two sets of dipole transitions [19-21]. The first two (lower energy pair) are the $1 s \rightarrow 4 p_{\pi}$ transition ( $4 p$ orbitals perpendicular to the $\mathrm{CuO}$ planes) and the second two $\mathrm{T}$ the $1 s \rightarrow 4 p_{\sigma}$ transition (in-plane $4 p$ orbitals). Figure 3 shows that the resonant enhancement is associated with the $1 s \leftrightarrow 4 p_{\pi}$

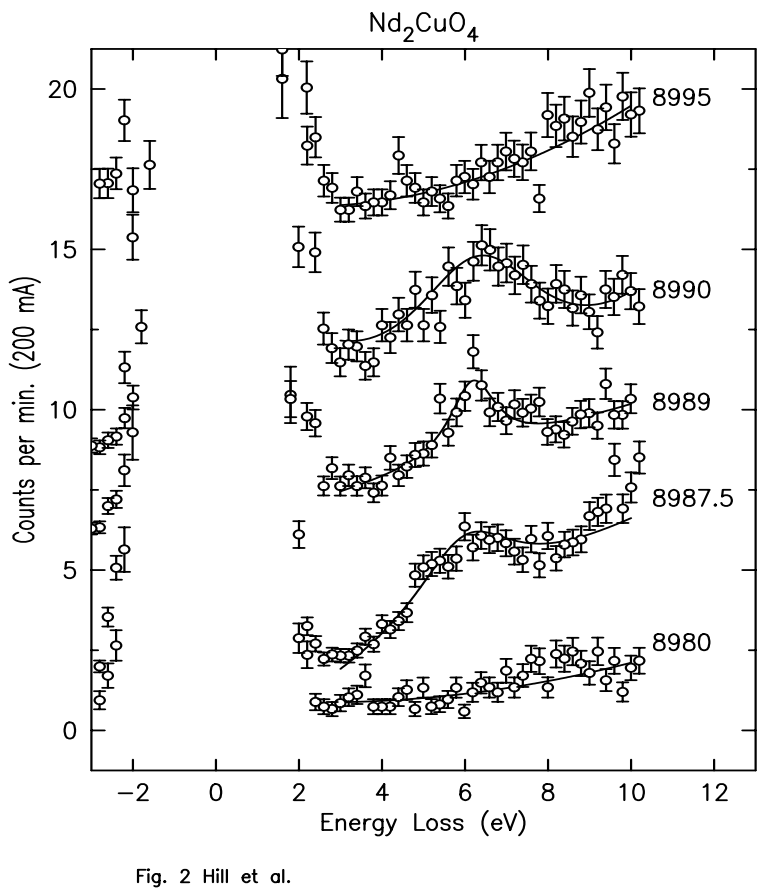

FIGURE 2. Scattered intensity as a function of energy loss $\left(E_{i}-E_{f}\right)$ for a number of incident energies in the vicinity of the $\mathrm{Cu} \mathrm{K}$-edge. Data are offset vertically for clarity and solid lines are guides to the eye. Figure taken from ref. [13]. 
transition. There was no apparent enhancement at the $1 s \leftrightarrow 4 p_{\sigma}$ transition. The absence of this latter transition is a result of the polarization dependence of the excitation process $\Gamma$ as discussed below.

In order to identify the character of the excitation $\Gamma$ numerical calculations of the electronic structure of $\mathrm{Nd}_{2} \mathrm{CuO}_{4}$ were performed within the Anderson impurity model [22123113]. The intermediate states of the scattering process were then calculated within the same model including on-site interactions between both the $4 p$ and the core hole $\left(U_{p c}=5 \mathrm{eV}\right)$ and the $4 p$ and $3 d \operatorname{states}\left(U_{d p}=3 \mathrm{eV}\right)$ and a finite $4 p_{\pi}$ bandwidth of $2 \mathrm{eV}$. The scattering was then treated as a coherent second order (dipole) process between these states [22]:

$$
F\left(\omega_{f}, \omega_{i}\right)=\sum_{f}\left|\sum_{n} \frac{<f|T| n><n|T| g>}{E_{g}+\omega_{i}-E_{n}-i \Gamma}\right|^{2} \delta\left(E_{g}+E_{i}-E_{f}-\omega_{f}\right),
$$

where $\mid g>$ is the ground state of the Anderson Hamiltonian with energy $E_{g} \Gamma$ and $\mid n>$ and $\mid f>$ are the intermediate and final states with energies $\Gamma E_{n} \Gamma$ and $E_{f} \Gamma$ respectively. $T$ is the dipole transition operator and $\Gamma$ is the lifetime of the core hole in the intermediate state.

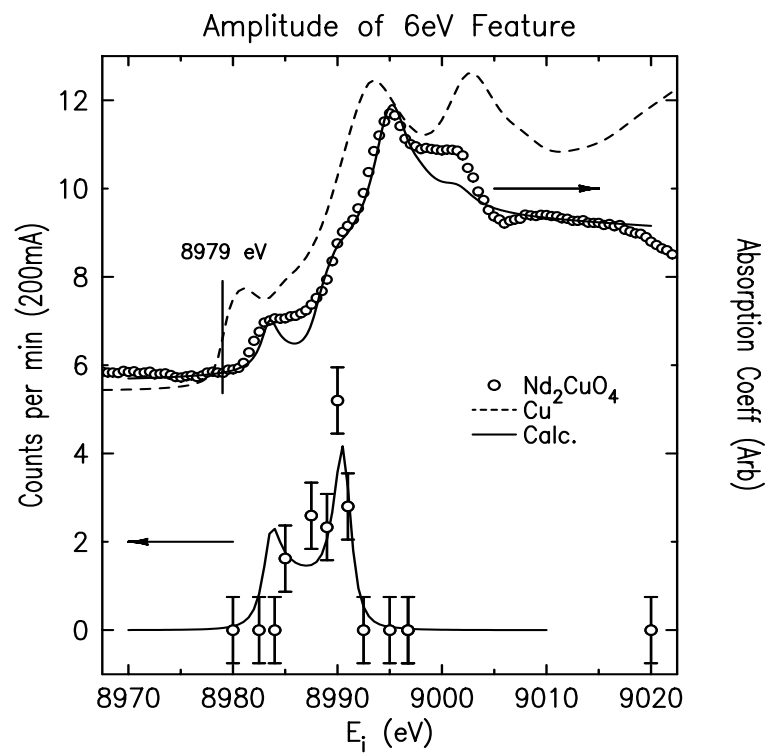

Fig. 3 Hill et al.

FIGURE 3. The amplitude of the $6 \mathrm{eV}$ excitation, as a function of incident energy, showing a resonance in the vicinity of $8990 \mathrm{eV}$ (open circles). The solid line is the result of the Anderson impurity model calculation described in the text. The top half of the figure displays the absorption measured for powdered $\mathrm{Nd}_{2} \mathrm{CuO}_{4}$ (open circles) together with a calculation using the same parameters as for the scattered intensity. The absorption from a $\mathrm{Cu}$ foil (dashed line) is shown for reference. Figure taken from ref. [13]. 
In the $\mathrm{CuO}$ planes $\Gamma$ the $\mathrm{Cu} 3 d^{9}$ configuration hybridizes with $3 d^{10} \underline{L} \Gamma$ where $\underline{L}$ represents an $\mathrm{O} 2 p$ ligand hole of finite bandwidth. Within the Anderson impurity model $\Gamma$ this results in discrete bonding and anti-bonding states composed of a mixture of $3 d^{9}$ and $3 d^{10} \underline{L}$ configurations $\Gamma$ with a continuous band between them (figure 4). The ground state is then the bonding state $\Gamma$ with about $60 \% 3 d^{9}$. The lowest edge of the continuous band (charge transfer gap) is about $2 \mathrm{eV}$ above this and the discrete anti-bonding state is $\sim 6 \mathrm{eV}$ above the ground state.

In the intermediate state of the resonant scattering process $\Gamma$ a $\mathrm{Cu} 1 \mathrm{~s}$ electron is excited to the (for example) $\mathrm{Cu} 4 p_{\pi}$ band $\Gamma$ and the core hole potential reverses the balance between the $3 d^{9}$ and $3 d^{10} \underline{L}$ configurations. The lowest energy state is then predominately $\underline{1 s} 3 d^{10} \underline{L} 4 p_{\pi}$ and is about $7 \mathrm{eV}$ lower than the anti-bonding state $\Gamma \underline{1 s} 3 d^{9} 4 p_{\pi}$ (fig. 4). These states form the 8983 and $8990 \mathrm{eV}$ features of the $\mathrm{Cu}$ K-edge XAS. (The $1 s \rightarrow 4 p_{\sigma}$ is similarly split.)

Energy loss spectra calculated within this model show a single excitation at 5.4 $\mathrm{eV} \Gamma$ when the incident photon energy is $8990 \mathrm{eV}$. (Note $\Gamma$ higher quality data taken at X25 placed the experimental value for the excitation at $5.7 \mathrm{eV})$. The peak results from the decay of the intermediate state into the anti-bonding excited state. A single peak is observed because $\Gamma$ for the $1 s 3 d^{9} 4 p_{\pi}$ intermediate state (which is excited at $8990 \mathrm{eV}$ ) there is no significant overlap with the continuous band $\Gamma$ which is predominately $3 d^{10} \underline{L}$. Decays into this band are therefore suppressed $\Gamma$ so that only

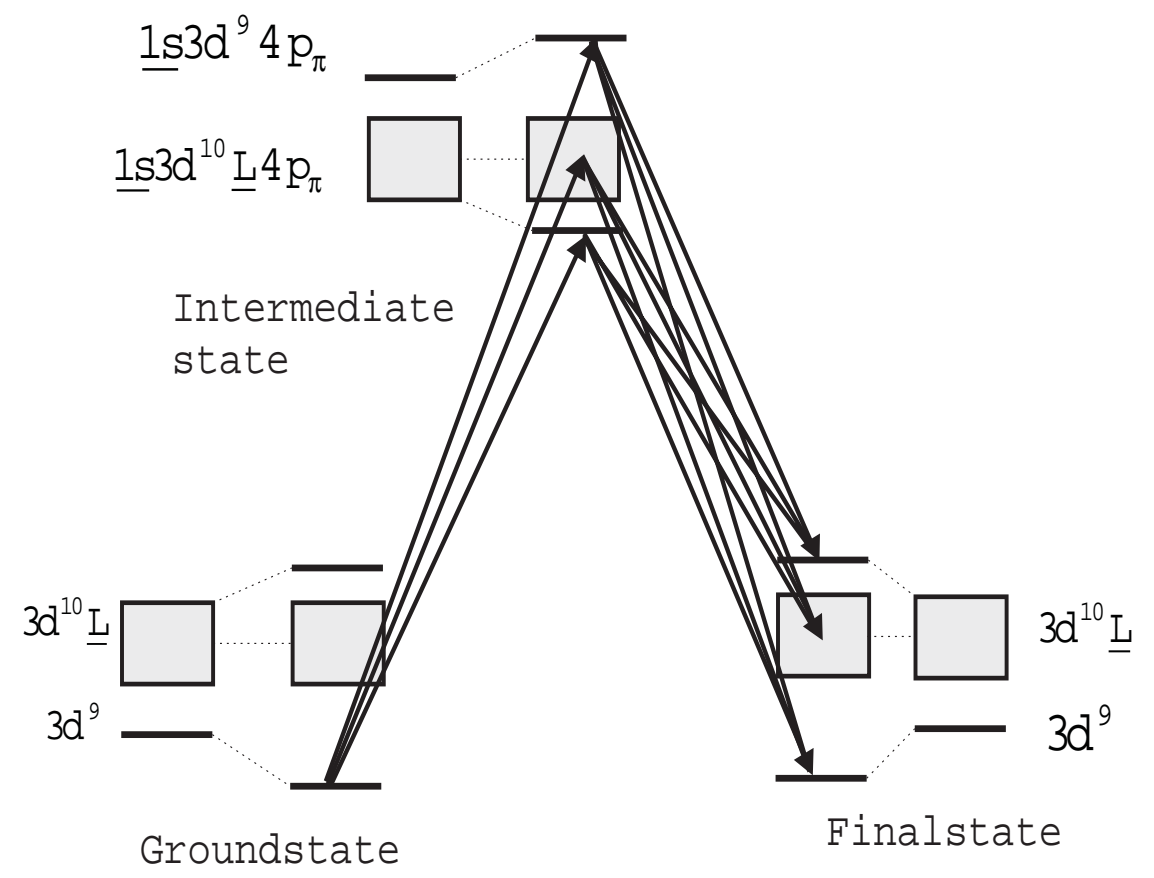

FIGURE 4. Schematic energy level diagram for inelastic scattering from a copper site. Arrows indicate processes summed over in the calculation of the scattered intensity. Figure taken from ref. [13]. 
the discrete anti-bonding state is observed. As the incident photon energy is moved away from $8990 \mathrm{eV} \Gamma$ the intensity of the $6 \mathrm{eV}$ feature decreases $\Gamma$ correctly reproducing the resonant behavior of this excitation. Thus $\Gamma$ the calculations showed good agreement with experimental observations in the vicinity of $8990 \mathrm{eV}$ and therefore the excitation was identified as a charge transfer excitation into an antibonding state.

In addition to $\mathrm{Nd}_{2} \mathrm{CuO}_{4}$ Ttwo other cuprates have also been studied $\mathrm{CuGeO}_{3}$ and $\mathrm{YBa}_{2} \mathrm{Cu}_{3} \mathrm{O}_{7}[14 \Pi 7] \Gamma$ and in each case $\Gamma$ similar excitations were observed $\Gamma$ at $6.5 \mathrm{eV}$ and $\approx 5$ eVTrespectively. The similarity in excitation energies suggests that the important structure in determining the electronic properties is the $\mathrm{CuO}_{4}$ plaquette $\Gamma$ which these three materials have in common. This is consistent with the charge transfer identification of the excitation.

\section{Polarization Dependence}

As noted above $\Gamma$ in the original work on $\mathrm{Nd}_{2} \mathrm{CuO}_{4} \Gamma$ there were no resonances associated with the higher energy transitions associated with the $4 p_{\sigma}$ band. In ref. [13] Гit was hypothesised that this was connected with polarization effects. In order

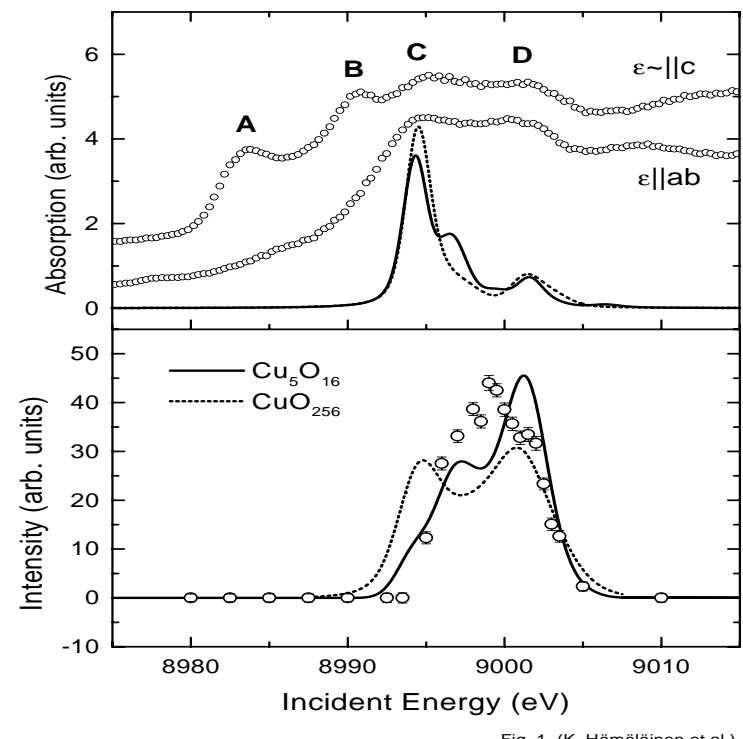

FIGURE 5. Top panel: Polarization dependent absorption measured via monitoring the fluorescence yield . Lower panel: The intensity of the $6 \mathrm{eV}$ excitation extracted by fitting the individual energy loss scans as a function of incident energy (open symbols). The solid and dashed lines in both panels are the results of numerical calculations for the $1 s \rightarrow 4 p_{\sigma}$ transitions described in the text. Figure taken from ref. [15]. 
to test this $\Gamma$ measurements were carried out with the incident polarization aligned in the $\mathrm{CuO}$ planes $(\epsilon \| a b$ plane $) \Gamma$ that is about $90^{\circ}$ to the earlier work $(\epsilon \approx \perp a b$ plane). The momentum transfer was perpendicular to the $\mathrm{CuO}$ sheets $(q \| c) \Gamma$ as previously [15П16].

As in the previous experiment a $6 \mathrm{eV}$ excitation is observed only at certain $\Gamma$ resonant $\Gamma$ incident energies. The amplitude of the $6 \mathrm{eV}$ excitation as a function of incident energy was extracted by fitting a series of scans with a Gaussian peak on a linear background. The resulting amplitudes are shown in the bottom half of Fig. 5 (open circles). The peak positions and widths were almost constant showing only very slight variations (in fact $\Gamma$ the fits were essentially unchanged if the peak position and width were held fixed). A single resonance was observed at 8999.5 $\mathrm{eV}$ Tof width $\approx 7 \mathrm{eV}$ FWHM. Thus $\Gamma$ in this geometry $\Gamma$ a resonant enhancement is observed at a $4 p_{\sigma}$ transition and not a $4 p_{\pi}$ excitation.

Polarized x-ray absorption spectraГshown in top half of Fig. 5 Pprovide a natural explanation for these results. These data were obtained by monitoring the intensity of the $\mathrm{Cu} K \alpha$ fluorescence from the sample as the incident energy was tuned through the $K$-edge and were not corrected for self absorption effects [24].

Features A and B (fig. 5) are associated with the transitions to the $4 p_{\pi}$ orbitals and they are preferentially excited when the incident polarization is along $c$ direction (top panelГfig 5) and aligned with the excited state orbitals $\Gamma$ maximizing the dipole matrix element for the excitation process. Conversely $\Gamma \mathrm{C}$ and $\mathrm{D}$ are associated with $1 s \rightarrow 4 p_{\sigma}$ transitions and $\Gamma$ correspondingly $\Gamma$ these were most prominent when the incident polarization is in the $a b$ plane and parallel with the $4 p_{\sigma}$ orbitals.

The significance of these data is that the final state of the XAS process is the intermediate state in the resonant scattering process. For a significant resonant enhancement to occur $\Gamma$ it is necessary that the intermediate state be strongly excited. The polarized XAS results show that the $4 p_{\sigma}$ intermediate states are strongly excited (and the excitation transitions $1 s \rightarrow 4 p_{\pi}$ strongly suppressed) when the polarization is in the $\mathrm{CuO}$ planes. It was this polarization dependence of the excitation process which was responsible for the absence of resonant enhancements at the $4 p_{\sigma}$ position in figure 3 and for the absence of a $4 p_{\pi}$ resonance in figure 5 .

A strong excitation to the intermediate state $\Gamma$ however $\Gamma$ is only a necessary condition for observing a resonant enhancement $\Gamma$ it is not a sufficient one. This is demonstrated by the absence of resonances associated with the $\underline{1 s} 3 d^{10} \underline{L}$ intermediate states (features $\mathrm{A}$ and $\mathrm{C}$ ) in either geometry. An explanation for this absence is presented in the following section.

\section{Resonant Lineshape and Non-Local Effects}

Calculations of electronic structure and the resonant inelastic x-ray scattering process $\Gamma$ based on a single copper site $\Gamma$ correctly predict the incident polarization dependence discussed above. However $\Gamma$ they fail to account for the systematic absence of the $\underline{1 s} 3 d^{10} \underline{L}$ resonances (features A and C); specifically in both cases 


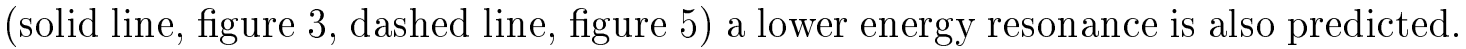
This failure of the single site calculation led to speculation [13] that the suppression of the lower energy resonance was due to non-local effects arising from the fact that the real system does not consist of a single isolated $\mathrm{CuO}_{4}$ cluster $\mathrm{Cbut}$ rather forms a continuous $\mathrm{CuO}$ network $\Gamma$ and therefore more complicated $\Gamma$ solid state $\Gamma$ effects may be required to accurately describe the resonance process.

One explanation for the discrepancy came from non-local screening effects $\Gamma$ which are active in the case of a $3 d^{10} \underline{L}$ intermediate state $\Gamma$ of the type first proposed by

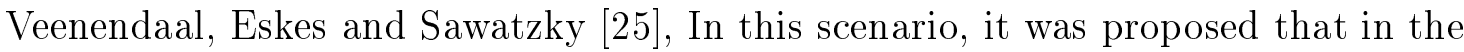
$3 d^{10} \underline{L}$ intermediate state $\Gamma$ the $\underline{L}$ hole is repelled by the $\underline{1 s}$ on the copper site and moves off elsewhere in the copper oxide sheet $\Gamma$ to form a Zhang-Rice singlet bound state [27] with a $3 d^{9}$ copper site. The binding energy of this singlet stabilizes such a process. This non-local intermediate state cannot then simply decay into the charge-transfer antibonding state (the "6 eV" feature) $\Gamma$ which is a local excitation; the transition probability between states with and without a Zhang-Rice singlet is small $\Gamma$ and thus no resonance would be observed. The solid line of fig. 3 is the result of calculations performed for a single copper site for which there are no such non-local effects $\Gamma$ since the $\underline{L}$ hole of necessity must remain in the immediate vicinity of the central core hole. This latter state has significant overlap with the antibonding state (and indeed also with the continuous band of states in fig 3) and therefore a resonance appears at $8983 \mathrm{eV}$ in the calculations.

In order to explore explicitly such non-local screening effects $\Gamma$ the earlier calculations were improved upon $\Gamma$ and the resonant inelastic scattering was modeled with a $\mathrm{Cu}_{5} \mathrm{O}_{16}$ cluster $\Gamma$ in which five $\mathrm{CuO}_{4}$ plaquettes were arranged in the $a b$ plane with $\mathrm{D}_{4 h}$ symmetry (see e.g. [26]). A Cu $3 d_{x^{2}-y^{2}}$ orbital was placed at each $\mathrm{Cu}$ site $\Gamma$ and an $\mathrm{O} 2 p_{x}$ or $2 p_{y}$ orbital at each $\mathrm{O}$ site hhybridized with the neighboring $\mathrm{Cu} 3 d_{x^{2}-y^{2}}$ orbital. Parameter values used in the cluster model were; the charge transfer energy $\Delta=2.5$ eV $\Gamma$ the electron hopping energy between neighboring $\mathrm{Cu} 3 d$ and $\mathrm{O} 2 p$ orbitals $\Gamma T_{p d}=1.21 \mathrm{eV} \Gamma$ that between the neighboring $\mathrm{O} 2 p$ orbitals $T_{p p}=0.55 \mathrm{eV} \Gamma$ the on-site Coulomb interaction between $\mathrm{Cu} 3 d$ electrons $U_{d d}=8.8 \mathrm{eV} \Gamma$ and for the $\mathrm{Cu} 1 s$ core hole potential acting on the $\mathrm{Cu} 3 d$ hole $U_{d c}=8.0 \mathrm{eV}$. For the $\mathrm{Cu}$ $4 p$ orbitals nearest neighbor hopping was considered $\Gamma$ as represented by the SlaterKoster parameters $(p p \sigma)=0.24 \mathrm{eV}$ and $(p p \pi)=-0.8 \mathrm{eV} \Gamma$ the on-site Coulomb interaction between $\mathrm{Cu} 4 p$ and $3 d$ states $U_{p d}=3.0$ eV $\Gamma$ and the on-site Coulomb potential of the core hole acting on the $4 p$ electron $-U_{p c}=-4.0 \mathrm{eV}$. The resonant inelastic spectra were again calculated with the coherent second order optical formula following an exact diagonalization of electronic states of the cluster.

The calculated result for the $6 \mathrm{eV}$ amplitude at the $1 s \rightarrow 4 p_{\sigma}$ transition is shown in the bottom panel of Fig. 5 (solid curve) Tafter broadening by incident and final resolutions of $2.0 \mathrm{eV}$. A strong resonance is seen only at the higher energy XAS feature $\left(\underline{1 s} 3 d^{9}\right)$ in broad agreement with the experimental results. For comparison $\Gamma$ the results of the Anderson impurity model calculation for a large cluster with a single $\mathrm{Cu}$ site and many $\mathrm{O}$ sites (256) are also shown (dashed curve). This exhibits resonances at both the $\underline{1 s} 3 d^{9}$ and $\underline{1 s} 3 d^{10} \underline{L}$ energies. Taken together $\Gamma$ these calcu- 
lations demonstrated that the suppression of the resonant enhancement associated with the $\underline{1 s} 3 d^{10} \underline{L}$ intermediate states results from the presence of multi-Cu sites and reflects non-local effects $\Gamma$ as originally speculated.

In addition $\Gamma$ the higher intensity obtained at the $4 p_{\sigma}$ resonance (at least partially due to the higher flux at beamline X25) allowed a more accurate determination of the excitation energy. It was found to be $E_{C T}=5.7 \mathrm{eV}$. The single site calculation estimated $E_{C T}=5.2 \mathrm{eV}$ and the multi-site calculation $\Gamma E_{C T}=5.7 \mathrm{eV}$. Thus it appears that non-local effects are also manifest in the position of the excitation as well as the incident energy dependence. Such quantitative comparisons highlight the potential for resonant inelastic x-ray scattering measurements to provide sensitive tests of electronic structure calculations for these materials.

The non-local hypothesis was also tested experimentally which the crystal structure is such that the connectivity of the $\mathrm{CuO}_{4}$ plaquettes is radically different. In this regard $\mathrm{CuGeO}_{3}$ provided a good test case since the $\mathrm{CuO}_{4}$ units are connected in a 1 dimensional "edge-sharing" chain in contrast to the two dimensional "corner-sharing" network of $\mathrm{Nd}_{2} \mathrm{CuO}_{4}$. If the non-local ideas are correct $\Gamma$ one would expect significant differences in the resonant lineshapes for the two materials.

The incident energy dependence of the inelastic scattering in $\mathrm{CuGeO}_{3}$ is shown in fig. 6 [together with the absorption as measured in a powder sample [14П17]. In line with the expectations outlined above $\Gamma$ the resonance lineshape was found to be

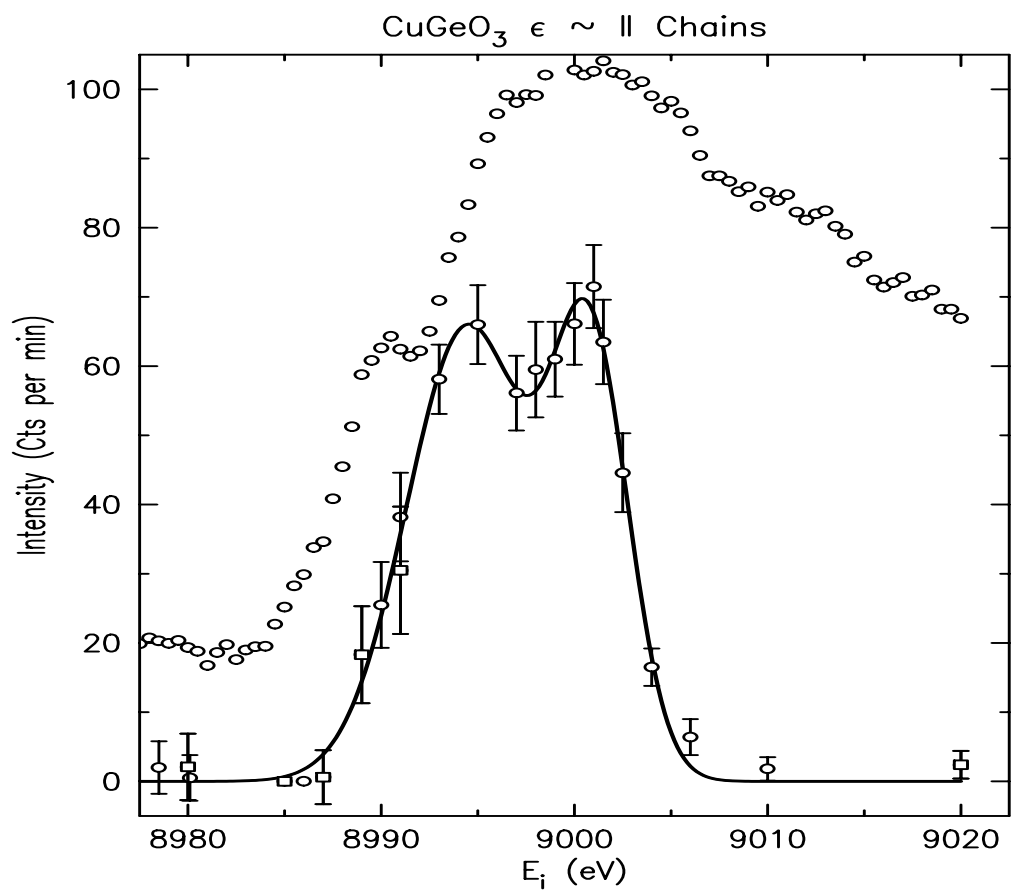

FIGURE 6. Incident energy dependence of the inelastic scattering in $\mathrm{CuGeO}_{3}$. Solid line is a guide to the eye. The absorption of powdered $\mathrm{CuGeO}_{3}$ is also shown. Figure taken from ref. [14]. 
quite different $\Gamma$ with a width three times that observed in $\mathrm{Nd}_{2} \mathrm{CuO}_{4}$ and a strong suggestion of two resonances being present. While these results have yet to be analyzed in the context of detailed electronic structure calculations $\Gamma$ they are certainly consistent with the idea that non-local effects are important in determining the resonance phenomena.

\section{E Momentum Dependence}

The $q$-dependence of the charge transfer scattering was also investigated. Data were taken in a vertical geometry $\Gamma$ i.e. with the polarization in the $a b$ plane $\Gamma$ and at the peak of the $4 p_{\sigma}$ resonance $\left(E_{1}=8999.5 \mathrm{eV}\right)$. The excitation was measured for momentum transfers in the range $3.5-7.9 \AA^{-1}$. The observed intensities were normalized by the intensity of the $K \beta_{1}$ emission line $\Gamma$ as measured with the incident photon energy set well above the edge $\Gamma$ to account for any variations in the beam footprint at different scattering angles. No dispersion in the peak position $\Gamma$ width $\Gamma$ or intensity was observed.

As emphasized above $\Gamma$ this charge transfer excitation is a localized excitation and thus would not be expected to exhibit much dispersion. It was therefore not possible to comment from these data on the speculations of ref.s [28I29] that q-dependent information can be obtained with the resonant technique. However $\Gamma$ these data do rule out any strong angular intensity dependence in the resonant cross-section in this geometry. Future work will focus on the $2 \mathrm{eV}$ charge transfer gap excitation for which predictions of dispersion have been made [18].

\section{OTHER WORKS}

Finally there has been recent work on other copper oxides $\Gamma$ namely that of $\mathrm{Ab}$ bamonte et al. [28] on $\mathrm{La}_{2} \mathrm{CuO}_{4}$ and $\mathrm{Sr}_{2} \mathrm{CuO}_{2} \mathrm{Cl}_{2}$. These experiments were carried out at X21 and at 3-ID an undulator beamline at the Advanced Photon Source. Broadly the results obtained in these materials are consistent with those discussed above. In particular $\Gamma$ a feature was observed around $6 \mathrm{eV}$ which is attributed to charge transfer excitations between the copper and oxygen. In addition $\Gamma$ the $\mathrm{Sr}_{2} \mathrm{CuO}_{2} \mathrm{Cl}_{2}$ experiments were performed at $0.45 \mathrm{eV}$ resolution and were able to resolve a feature at $2 \mathrm{eV}$ energy loss $\Gamma$ which was attributed to the charge transfer gap (that is to transitions to the bottom of the continuous band

However in contrast to the approach outlined above $\Gamma$ these authors chose to analyze their data following the ideas put forth by Platzman and Isaacs [29]. Specifically $\Gamma$ they follow a perturbative approach in treating the core hole-valence electron interaction and argue that near a sharp dipole allowed transition $\Gamma$ the dominant term occurs in third order and has the form $\Gamma$

$$
M=\sum_{\underline{1 s, 4 p}} \frac{M_{e m} M_{C o u l} M_{a b s}}{\left(\omega_{f}-E_{\underline{1 s}, 4 p}+i \gamma_{K}\right)\left(\omega_{i}-E_{\underline{1} s, 4 p}+i \gamma_{K}\right)}
$$


Where $\mathrm{M}_{e m}\left(\mathrm{M}_{\text {abs }}\right)$ are the dipole emission (absorption) matrix elements $\Gamma \mathrm{M}_{\text {Coul }}$ is the Coulomb interaction between the core and valence states $\Gamma \mathrm{E}_{\underline{1 s}, 4 p}$ is the energy of the virtual exciton-like intermediate state and $\gamma_{K}$ is its lifetime. Physically $\Gamma$ this expression represents a "shakeup" process $\Gamma$ whereby a virtual $\underline{1 s} 4 p$ exciton is created $\Gamma$ scatters off the valence electrons $\Gamma$ leaving them in an excited state $\Gamma$ and decays. By assuming that all the intermediate states are degenerate $\Gamma$ of energy $E_{K} \Gamma$ then the energy denominators can be pulled out of the sum leaving

$$
\frac{d \sigma}{d \Omega d \omega} \propto \frac{S_{K}(\mathbf{q}, \omega)}{\left.\left[\left(\omega_{f}-E_{K}\right)^{2}+\gamma_{K}^{2}\right]\left[\left(\omega_{i}-E_{K}\right)^{2}+\gamma_{K}^{2}\right)\right]}
$$

with

$$
S_{K}(\mathbf{q}, \omega)=\frac{2 \pi}{\hbar} \sum_{f}\left|\sum_{\underline{1 s}, 4 p} M_{e m} M_{\text {Coul }} M_{a b s}\right|^{2} \delta\left(\omega-E_{f}+E_{i}\right)
$$

The quantity $\Gamma \mathrm{S}_{K}(\mathbf{q}, \omega)$ depends only on the difference quantities $\mathbf{q}=\mathbf{k}_{\mathbf{i}}-\mathbf{k}_{\mathbf{f}}$ and $\omega=\omega_{i}-\omega_{f}$. Since it does not depend explicitly on the incident energy $\Gamma$ it is a fundamental property of every spectrum. Abbamonte et al. show that their $\mathrm{La}_{2} \mathrm{CuO}_{4}$ data exhibit the correct scaling predicted by such arguments $\Gamma$ for a reasonable choice of $E_{K}$ and $\gamma_{K}$. They further show that $\mathrm{S}_{K}(\mathbf{q}, \omega)$ is related to a particular weighted sum of $\mathrm{S}(\mathbf{q}+\mathbf{G}, \omega)$ where $\mathrm{S}(\mathbf{q} \omega)$ is the dynamic structure factor of the valence electrons.

Note one of the features of equation (3) is that it predicts that the position of the energy loss feature will depend on the incident excitation energy. Such dispersion was seen in the $\mathrm{La}_{2} \mathrm{CuO}_{4}$ data (and perhaps in the $\mathrm{NiO}$ work [2]) $\Gamma$ but not in the $\mathrm{Nd}_{2} \mathrm{CuO}_{4}$ and $\mathrm{CuGeO}_{3}$ experiments. However $\Gamma$ the size of the dispersion is a sensitive function of the parameters and small changes in $\Gamma$ for example the excitation lifetime $\Gamma$ can greatly reduce the any dispersion observed in the energy loss feature.

It remains to be seen under what conditions the approximations used to obtain equation (3) are valid. If it is generally applicable $\Gamma$ the importance of the ability to relate measured quantities to the dynamic structure factor cannot be overstated.

\section{SUMMARY}

Recent progress in the use of resonant inelastic x-ray scattering in the hard x-ray regime to study transition metal oxides was reviewed. Particular emphasis was placed on the work on $\mathrm{Nd}_{2} \mathrm{CuO}_{4}$ in which the details of the resonant process was explored. A charge transfer excitation to the antibonding excited state is seen at $5.7 \mathrm{eV}$ when the incident photon energy is tuned to certain resonances in the vicinity of the copper K-edge. It was shown that the systematic absence of resonant enhancements associated with certain transitions is caused by the polarization dependence of the excitation process into the intermediate state. Specifically the 
relative orientation of the incident polarization and the particular $4 p$ intermediate state. In addition $\Gamma$ in order that there be a resonant enhancement of the inelastic signal $\Gamma$ there needs to be a significant overlap between the intermediate states and the particular excited state being studied. In the case of the antibonding excitation in $\mathrm{Nd}_{2} \mathrm{CuO}_{4} \Gamma$ this was manifest in the absence of any resonant enhancement at transitions corresponding to well screened intermediate states. Experiments on other materials and calculations are consistent with explanations based on these intermediate states being extended $\Gamma$ non-local objects with little overlap with the localized excitation being studied. These results suggest that such experiments may provide a sensitive measurement of the breadown of the localized picture of the electron structure $\Gamma$ and of the resulting importance of non-local effects.

The important remaining question in the field concerns the interpretation of the inelastic spectra themselves $\Gamma$ as measured on resonance $\Gamma$ and in particular what $\Gamma$ if any connection can be made to the dynamic structure factor of the valence electrons. In this regard $\Gamma$ q-dependent measurements to be carried out in the near future will be very important in answering such questions.

\section{ACKNOWLEDGEMENTS}

I would particularly like to acknowledge the contributions of C.-C. Kao $\Gamma$ with whom all of this work was performed. In addition it is a great pleasure to acknowl-

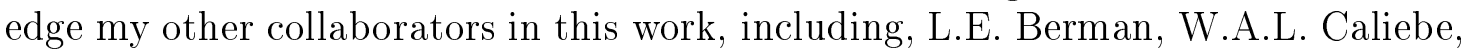

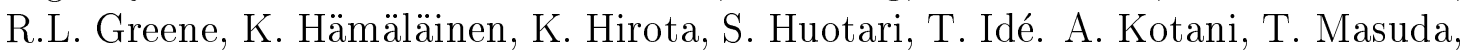

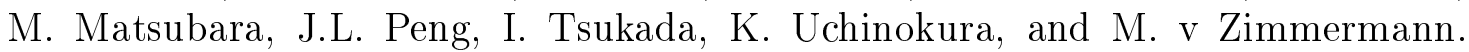
This work was supported by the U.S. Department of EnergyГDivision of Materials Science under contract no. DE-AC02-98CH10886.

\section{REFERENCES}

1. W. Schülke Handbook on Synchrotron Radiation, Ed.s D.E. Moncton, and G.S. Brown, North Holland, 1991, pp 565-639.

2. C.-C. Kao, W.A.L. Caliebe, J.B. Hastings and J.M. Gillet, Phys. Rev. B 54, 16361 (1996).

3. P. Eisenberger, P.M. Platzman, and H. Winick, Phys. Rev. B 13 2377, (1976).

4. Y. Ma et al., Phys. Rev. Lett., 69, 2598 (1992).

5. J.J. Jia, T. A. Callcott, E. L. Shirley, J.A. Carlisle, L.J. Terminello, A. Asfaw, E.L. Ederer, F.J. Himpsel and R.C.C. Perera, Phys. Rev. Lett., 76, 4054 (1996).

6. S.M. Butorin et al., Phys. Rev. Lett. 77, 574 (1996).

7. S.M. Butorin et al., Phys. Rev. B 55, 4242 (1997).

8. P. Kuiper, J.-H. Guo, C. Sathe, L.-C. Duda, J. Nordgren, J.J.M. Potuizen, F.M.F. de Groot, and G.A. Sawatzky, Phys. Rev. Lett. 80, 5204 (1998).

9. A. Moewes, S. Stadler, R.P. Winarksi, D.L. Ederer, M.M. Grush and T.A. Callcott , Phys. Rev. B. 58, R15951 (1998). 
10. F. Gel'mukhanov and H. Agren, Phys. Rev. B 49, 4378, (1994).

11. M. v Veenendaal and P. CarraГPhys. Rev. Lett.Г78Г2839 (1997).

12. E. ShirleyГPhys. Rev. Lett.Г80Г794 (1998).

13. J.P. HillГC.-C. KaoГW.A.L. CaliebeГM. MatsubaraГА. KotaniГJ.L. Peng and R.L. GreeneГPhys. Rev. Lett. 80Г4967 (1998).

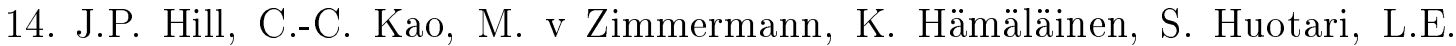

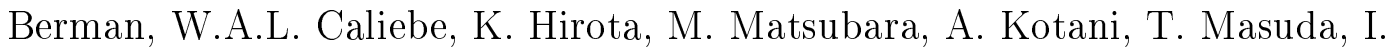
Tusukada and K. UchinokuraГJ.L. Peng and R.L. GreeneГ to appear in Jap. J. Appl. Phys. (1999).

15. K. HämäläinenГJ.P. HillГS. HuotariГC.-C. KaoГL.E. BermanГA. KotaniГJ.L. Peng $\Gamma$ and R.L. GreeneГsubmitted to Phys. Rev. B.

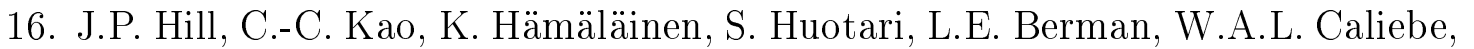

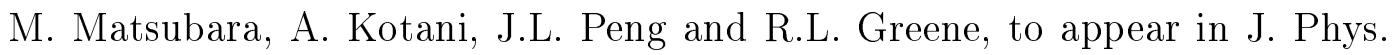
Chem. of Solids. (1999).

17. J.P. HillГC.-C. KaoГM. v ZimmermannГK. HirotaГА. KotaniГT. MasudaГI. Tusukada and K. Uchinokura Tin preparation

18. K. TsutsuiГT. Tohyama and S. MaekawaГLANL Cond. Mat/9905372

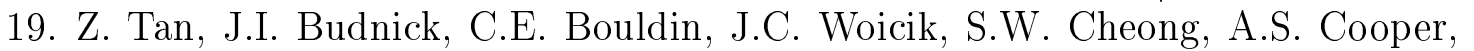
G.P. Espinoza and Z. FiskГPhys. Rev. B 42 Г1037 (1990).

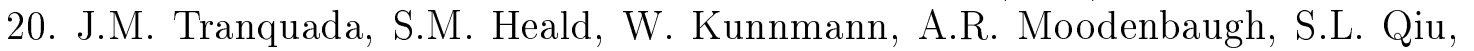

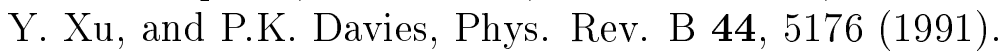

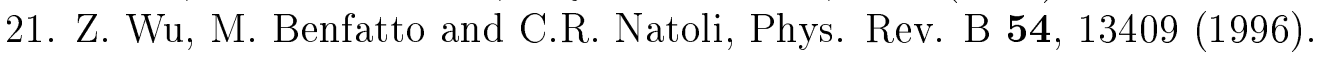

22. S. TanakaГK. Okada and A. KotaniГJ. Phys. Soc. Jpn. 60Г3893 (1991).

23. S. Tanaka and A. KotaniГJ. Phys. Soc. Jpn. 62Г464 (1993).

24 . Note that while the sample geometry made it possible to place the incident polarization solely in the $a b$ plane (vertical scattering geometry) $\Gamma$ in the horizontal scattering geometry the polarization was in fact $30^{\circ}$ off the $c$ direction.

25. M.A. VeenendaalГH. Eskes and G.A. Sawatzky ГPhys. Rev B. 47Г11462 (1993).

26. K. Okada and A. KotaniГ J. Electron Spectrosc. Relat. Phenom. 86Г 119 (1997).

27. F. Zhang and T. RiceГPhys. Rev. В 37Г3759 (1988).

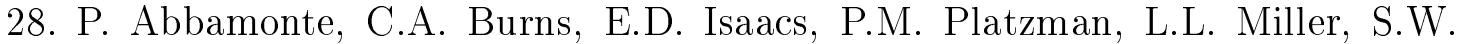
CheongГM.V. KleinГPhys. Rev. Lett.Г83Г860 (1999).

29. P.M. Platzman and E.D. IsaacsTPhys. Rev. B. 57Г11107 (1998). 